\title{
Actualización: \\ Diagnóstico de demencia leve en atención primaria
}

Diagnosis of mild dementia in primary care

Gabriel Villalón*

\section{Resumen}

A medida que la población envejece la demencia y su principal motivo de consulta, la queja cognitiva, se tornan problemas de salud cada vez más frecuentes. El médico de atención primaria suele encontrar obstáculos a la hora de realizar con eficiencia el diagnóstico de esta entidad, sobre todo en los estadios iniciales de la misma.

En la presente actualización se aborda en particular una de ellas: la selección de un instrumento práctico, fiable y disponible para objetivar el déficit cognitivo sospechado a través de la valoración clínica. Asimismo se revisa la evidencia acerca de una prueba validada en nuestro medio, la evaluación cognitiva de Addenbrooke's, que podría servir de alternativa a este fin en el consultorio de atención primaria.

\section{Abstract}

As the population ages, dementia, and cognitive complaints (its main reason for consultation) becomes increasingly common health problems. The primary care physician often finds obstacles to efficiently diagnose this entity, especially in its early stages. In this update, the selection of a practical, reliable and available instrument to identify the cognitive deficits that are suspected by clinical assessment is addressed. It also reviews the evidence for a locally validated test, the Addenbrooke's Cognitive Examination, which may serve as an alternative for diagnostic purposes in the primary care clinic.

Palabras clave: deterioro cognitivo, demencia leve, diagnóstico, pruebas de evaluación cognitiva, Addenbrooke's. Key words: cognitive impairment, mild dementia, diagnosis, cognitive test, Addenbrooke's.

Villalón G. Diagnóstico de demencia leve en atención primaria. Evid. actual. práct. Ambul; 12(2):66-71, Abril - Junio 2009.

Introducción: deterioro cognitivo y demencia como problemas de salud con impacto socio-sanitario creciente

Según datos provenientes de países desarrollados, la prevalencia promedio de demencia en pacientes de 60 a 85 años oscila entre el 1,15 a $22,8 \%$ respectivamente ${ }^{1}$

En Argentina y América Latina, esta información es más imprecisa. En un estudio piloto poblacional realizado en Cañuelas, provincia de Buenos Aires, se estimó una prevalencia global de deterioro cognitivo del $23 \%$, con valores que oscilaron entre un 16,9 entre los 60 y los 69 años y $42,5 \%$ para los mayores de $80^{2}$. A partir de estos datos se estima que en nuestro país existirían alrededor de 1.000 .000 de sujetos con deterioro cognitivo, y 480.000 con demencia**.

Datos de Latinoamérica indican que la prevalencia de demencia en mayores de 65 años oscila entre 2,9 y $23,8 \%{ }^{3}$.

Proyecciones para años venideros indican que estos índices aumentarán a nivel global; previéndose la duplicación de la prevalencia de demencia cada 20 años ${ }^{1}$. Esto probablemente se asocie al incremento de la población añosa y de la esperanza de vida.

La enfermedad de Alzheimer (DA) y otras demencias representan una carga significativa de enfermedad. Esto se traduce para la persona afectada, en años vividos con una discapacidad y años potenciales de vida perdidos. El índice que mejor resume lo anterior se conoce como DALY (Disability Adjusted Life Years, o años de vida ajustados por discapacidad) y se expresa en años. Según datos de la Organización Mundial de la Salud, en 2005 las enfermedades neurológicas dieron cuenta del $6,3 \%$ de los DALY a nivel mundial. De éstos, las enfermedades cerebrovasculares representaron poco más del $50 \%$, y la enfermedad de Alzheimer, junto al Parkinson y otras demencias, el $13 \%{ }^{3}$

Como puede sospecharse, la demencia impacta de manera significativa sobre los costos de salud, tanto a nivel familiar como socio sanitario. En nuestro país se llevó a cabo un pequeño estudio que evaluó el impacto económico de las demencias. Teniendo en cuenta los costos directos (aquellos provenientes de pagos de prestaciones médicas, cuidadores e institucionalizaciones) y los indirectos (pérdida de ingresos del paciente y/o la familia derivados del cuidado) se concluyó que esta enfermedad multiplica en 4,8 y 8,8 veces el gasto en salud de pacientes con demencia no institucionalizados e institucionalizados, respectivamente, comparado con el mismo gasto en pacientes de la misma edad pero sin demencia. La mayor parte de estos costos son asumidos por la familia ${ }^{3}$.

Para los médicos de atención primaria que atienden pacientes añosos, el abordaje de las consultas por fallas mnésicas (principal forma de presentación temprana de los síndromes demenciales) constituye todo un desafío. La complejidad del proceso diagnóstico, la falta de familiaridad con las herramientas de evaluación cognitiva, y las dificultades con el manejo del tiempo de la consulta médica podrían explicar por qué solo son diagnosticados menos del $25 \%$ de los casos de demencia tipo Alzheimer en Canadá 6 .

Si bien la evidencia actual es aun insuficiente para expedirse a favor o en contra del rastreo de demencia en la población general asintomática (recomendación grado I de la fuerza de tareas preventiva de EE.UU ó USPSTF $)^{5}$ su diagnóstico temprano ("case finding") podría resultar útil por algunas razones. Por un lado existe una pequeña proporción de trastornos reversibles que se presentan como deterioro cognitivo, cuyo diagnóstico diferencial no debe pasarse por alto. Cada vez son más los tratamientos sintomáticos disponibles para el manejo de las demencias, así como las diferentes estrategias de abordaje para sus distintas etiologías. También hay que tener presente que la reorganización de las familias de pacientes con demencia es más eficiente cuando ésta se diagnostica en forma temprana ${ }^{6}$. Todo esto, sumado al hecho de que la población anciana que consulta por problemas de memoria aumentará -y gran parte de ella estará a cargo de médicos de atención primaria- refuerza la necesidad que los médicos de cabecera actualicen sus competencias en el diagnóstico y manejo de este problema de salud.

\section{Camino al diagnostico de demencia}

El abordaje diagnóstico general de los pacientes que consultan por fallas mnésicas implica los siguientes pasos: realizar un

* Médico especialista en Medicina Familiar. Servicio de Medicina Familiar y Comunitaria del Hospital Italiano de Buenos Aires. gabriel.villalon@ hospitalitaliano.org.ar

** El deterioro cognitivo constituye un término mas amplio e impreciso, que puede abarcar desde el deterioro cognitivo leve, hasta los estadios avanzados de demencia. Se estima que aproximadamente un $50-60 \%$ de los pacientes con deterioro cognitivo cumplen con criterios de demencia. 
adecuado interrogatorio del paciente, sus familiares cercanos y/o cuidadores; llevar a cabo un adecuado el examen físico; administrar apropiadamente algunas pruebas cognitivas; y discriminar los diagnósticos diferenciales relevantes (a través de estudios complementarios) en los casos en que se plantee el diagnostico de demencia ${ }^{6}$.

En la actualidad la definición del DSM IV constituye el estándar de referencia para establecer el diagnóstico de demencia. Así, se considera la existencia de demencia ante un individuo que presente alteraciones de la memoria, sumado a la alteración de uno o más dominios cognitivos, a saber: agnosia (dificultad para reconocer objetos), apraxia (dificultad para realizar ciertas actividades motoras), afasia (trastorno del lenguaje) o trastornos ejecutivos (alteración en la capacidad de planificación de una conducta compleja). Estos deben ser lo suficientemente significativos como para causar un deterioro funcional objetivable ${ }^{6,7}$.

El diagnóstico de demencia es eminentemente clínico. Su "test de referencia"* está dado en la actualidad por la valoración clínica (compatible con los criterios anteriormente señalados) sustentada mediante la evaluación cognitiva realizada a través de baterías neuropsicológicas. Estas últimas consisten en una serie de pruebas complejas que habitualmente requieren de un tiempo considerable y personal especializado para su administración e interpretación. Son costosas y como puede sospecharse, pueden no estar fácilmente disponibles. Sin embargo, tales pruebas no necesariamente deben llevarse a cabo en la evaluación de todos los casos de demencia. De acuerdo con las recomendaciones del Tercer Consenso Canadiense en el Diagnostico y Tratamiento de la Demencia, las pruebas neuropsicológicas pueden ser de utilidad para distinguir el envejecimiento normal del deterioro cognitivo leve o los estadios leves de demencia; para valorar el riesgo de progresión del deterioro cognitivo leve a demencia; y para determinar los diagnósticos diferenciales de demencia y otros síndromes de deterioro cognitivo ${ }^{6}$.

En este contexto han ganado terreno cada vez más las pruebas breves de evaluación cognitiva. La más difundida y utilizada sigue siendo el Mini Mental State Examination (MMSE). Esta prueba requiere poco entrenamiento para su uso y puede administrarse en diez minutos. Si bien goza de alta sensibilidad y especificidad para discriminar entre los estadios moderados de demencia y la "normalidad", su utilidad para diferenciar los estadios leves es limitada, por lo que el diagnóstico de demencia no puede excluirse ante un resultado normal.

Estas limitaciones condujeron a confeccionar otras pruebas, o adaptar el MMSE con el objetivo de mejorar su rendimiento diagnostico de los estadios iniciales de la demencia (p. ej. Modified Mini Mental State Examination, test del reloj, Montreal Cognitive Asessment [MoCA test], DemTect, 7-minute Screen, General Practitioner Assessment of Cognition, entre otras) manteniendo al mismo tiempo las premisas de brevedad y practicidad en su administración. Sin embargo aun habiéndose logrado estos objetivos en algunos casos, no se puede recomendar una prueba por sobre otra. Ver tabla 1.

Tabla 1: características operativas de algunos instrumentos cognitivos breves para el diagnóstico de demencia

\begin{tabular}{|c|c|c|c|c|c|c|}
\hline \multirow[t]{2}{*}{ Instrumento } & \multirow{2}{*}{$\begin{array}{c}\text { Tipo de muestreo, } \\
\text { escenario } \\
y \text { país }\end{array}$} & \multirow[b]{2}{*}{ Punto de corte } & \multirow[b]{2}{*}{ LR+ (IC95\%)* } & \multirow[b]{2}{*}{ LR- (IB95\%) $)^{*}$} & \multicolumn{2}{|c|}{$\begin{array}{l}\text { VPP (VPV)* según las } \\
\text { prevalencias teóricas }\end{array}$} \\
\hline & & & & & $5 \%$ & $30 \%$ \\
\hline MMSE & $\begin{array}{l}\text { Cons ecutiva, atención primaria, } \\
\text { Australia }\end{array}$ & 24 & $3,4(2,6$ a 4,4$)$ & $0,26(0,17$ a 0,76$)$ & $15,2(98,7)$ & $59(90)$ \\
\hline Modified MMSE & $\begin{array}{l}\text { Aleatoria, poblacional, } \\
\text { Canadá }\end{array}$ & 77 & $8,6(7,0$ a 11,0$)$ & $0,14(0,07$ a 0,3$)$ & $31,2(99,3)$ & $78,7(94,3)$ \\
\hline Test del Reloj & $\begin{array}{l}\text { Cons ecutiva, especialistas, } \\
\text { Israel }\end{array}$ & $11 \gamma$ & $7,8$ (2,5 a 22$)$ & $0,17(0,1$ a 0,29 & $29,1 \quad(99,1)$ & $77(93,2)$ \\
\hline MoCA Test & $\begin{array}{l}\text { Conveniencia, mixto, } \\
\text { EEUU }\end{array}$ & 25 & $7,7(4,4$ a 12,7$)$ & $0,01(0,0$ a 0,61$)$ & $28,8(99,9)$ & $76,7 \quad(99,6)$ \\
\hline 7-Minute Screen & $\begin{array}{l}\text { Cons ecutiva, atención primaria, } \\
\text { EEUU }\end{array}$ & $\begin{array}{c}\text { Regresión } \\
\text { logstica }\end{array}$ & 47 (3 a 730) & $0,09(0,01$ a 0,59$)$ & $71,2(99,5)$ & $95,3(96,3)$ \\
\hline $\begin{array}{l}\text { General Practitioner } \\
\text { Assesment of Cognition }\end{array}$ & $\begin{array}{l}\text { Cons ecutiva, especialistas, } \\
\text { Australia }\end{array}$ & 10 & $4,8(3,5$ a 6,6$)$ & $0,22(0,14$ a 0,41$)$ & $20,2(98,9)$ & $67,3(91,4)$ \\
\hline
\end{tabular}

Modificado de Holsinger T, y col. ${ }^{7}$ Se tomaron a modo de referencia sólo algunos resultados. Sin embargo cabe mencionar que para una misma prueba pueden observarse variaciones significativas en las características operativas entre distintos trabajos ${ }^{\vee}$ Método Fiedman. LR: coeficiente de probabilidad, VPN/VPP: valores predictivos negativos y positivos ${ }^{*}$

Cuando se desea utilizar una determinada prueba diagnóstica, idealmente deberían seleccionarse aquellas que mejor se adapten al contexto donde se pretenden aplicar. Es por ello que a continuación comentaremos un artículo de validación diagnóstica realizado en Argentina, sobre una prueba breve de evaluación cognitiva. Esta podría resultar relevante por el contexto local de su aplicación.

\section{La evaluación cognitiva de Addenbrooke's (validada al español) ${ }^{8}$}

Objetivo

Validar al español la evaluación cognitiva de Addenbrooke's
(Addenbrooke's Cognitive Examination, o ACE) para la detección de demencia en una población que habla el idioma castellano (con buen nivel educativo). Como objetivo adicional se planteó estudiar la utilidad del coeficiente VLOM (ver comentario) para diferenciar la demencia tipo Alzheimer de la demencia frontotemporal (DFT).

\section{Métodos}

Se trata de un estudio de validación diagnóstica. Se llevo a cabo en Buenos Aires, Argentina. Incluyo 128 pacientes del Servicio de Neurología Cognitiva, Neuropsicología y Neuropsiquiatría del FLENI. Los participantes se seleccionaron mediante una revisión consecutiva de 300 historias clínicas. Se incluyeron aquellos pacientes que cumplieron con el diagnósti- 
co probable de DA según los criterios del NINCS-ADRDA (54); y pacientes con diagnóstico probable de DFT, según criterios de Lund y Manchester (22). Los pacientes debían presentar un grado leve de demencia (Clinical Dementia Rating o CDR 0,51). También se incluyó un grupo control (52 individuos) constituido por familiares de pacientes o voluntarios sanos apareados por edad, sexo y nivel de educación. Fueron excluidos los pacientes con criterios de depresión, patología cerebrovascular concomitante, antecedentes de adicción o abuso de sustancias y presencia de otras causas de deterioro cognitivo. La división entre pacientes con demencia y los controles se realizó según criterios del DSM-IV.

\section{Intervención y medición de resultados principales}

Todos los pacientes fueron sometidos al ACE y al CDR. Como evaluación independiente, los participantes fueron evaluados a través de una batería neuropsicológica y neuropsiquiátrica estándar; que junto al consenso clínico entre neurólogos, neuropsiquiatras y neuropsicólogos, constituyeron el test de referencia* para el diagnóstico de demencia. Como evaluación adicional, los pacientes con demencia fueron evaluados con RMN, SPECT (en los casos de DFT) y análisis de sangre completos, para excluir otras causas de deterioro cognitivo.

Los evaluadores estuvieron ciegos respecto de los antecedentes de cada paciente y sus respectivas pruebas. Se utilizaron curvas $\mathrm{ROC}^{*}$ para determinar los puntos de corte con mejores características operativas (sensibilidad, especificidad, valores predictivos positivos y negativos*) para el ACE y para la puntuación del Mini Mental State Examination (MMSE); y para comparar sus capacidades de discriminación según diferentes prevalencias teóricas.

\section{Resultados principales}

La edad promedio osciló entre 69 y 73 años y los años de educación entre 11,06 y 12,33 años. Tanto estas variables, como el sexo de los pacientes estuvieron balanceados en los tres grupos. El punto de corte elegido del ACE, con las mejores características operativas, fue 86 . Ver tabla 2.

Las curvas ROC favorecieron al ACE sobre el MMSE, con áreas bajo la curva de 0,984 y 0,908 respectivamente $(p<0,01)$. Los pacientes con DA mostraron puntajes promedio significativamente menores en los siguientes dominios: orientación, memoria total, aprendizaje y recuerdo de nombre y dirección, y habilidades visuo-espaciales.

Para el coeficiente VLOM, se estableció como punto de corte 4,12 . Con este valor se logra una sensibilidad ${ }^{*}$ de $95,5 \%$ (IC95\% 77 a 99) y una especificidad* de $40,7 \%$ (IC95\% 27 a 55) para DFT. Estableciendo como valores limítrofe 1,82 y 4,87 se lograría $100 \%$ de especificidad para DFT para valores por debajo del primero, y $100 \%$ de especificidad para DA para valores por encima del último, quedando un intervalo de incertidumbre diagnóstica en los valores intermedios.

Tabla 2: características operativas del Examen Cognitivo de Addenbrooke y del Examen "Minimental".

\begin{tabular}{|c|c|c|c|c|c|c|c|c|c|}
\hline \multirow[b]{2}{*}{ Instrumento } & \multirow[b]{2}{*}{ Sens* } & \multirow[b]{2}{*}{ Esp* } & \multirow[b]{2}{*}{ Esp* } & \multirow[b]{2}{*}{ LR+ (IC95\%)* } & \multirow[b]{2}{*}{ LR- (IC95\%) * } & \multicolumn{4}{|c|}{ VPP (VPN)* según las prevalencias teóricas } \\
\hline & & & & & & $5 \%$ & $10 \%$ & $20 \%$ & $30 \%$ \\
\hline \multirow[t]{2}{*}{ ACE } & $86 \pm$ & 92 & 96 & $23(8,7-60)$ & $0,08(0,04-0,16)$ & $55(99)$ & 72 & 85 & $91(97)$ \\
\hline & $68 \S$ & 90 & 86 & $6,4(3,9-10,4)$ & $0,11(0,06-0,21)$ & $25(99)$ & 42 & 62 & $73(95)$ \\
\hline \multirow[t]{2}{*}{ MME } & $27 \pm$ & 78 & 98 & $39(9,8-154,3)$ & $0,22(0,15-0,32)$ & $68(99)$ & 82 & 91 & $94(91)$ \\
\hline & $24 \S$ & 62 & 96 & $15,5(5,8-40,9)$ & $0,39(0,30-0,51)$ & $45(98)$ & 63 & 79 & $87(85)$ \\
\hline
\end{tabular}

\pm Versión argentina en pacientes con mayor nivel educativo. §Versión Española en pacientes con menor nivel educativo (ver comentario).

\section{Conclusión}

La versión al español del ACE es una herramienta de adecuada sensibilidad (92\%) para la detección de demencia en una población de alto nivel educativo. El coeficiente VLOM del ACE en español permite una orientación para el diagnostico diferencial entre DA y DFT.

Fuente de financiamiento: no reportada

\section{Comentarios}

EI ACE fue desarrollado originalmente en la Universidad de Addenbrooke, en Cambridge ${ }^{9}$. Esta prueba evalúa seis dominios cognitivos, con una puntuación máxima de 100: orientación (10), atención (8), memoria (35), fluencia verbal (14), lenguaje (28) y habilidades visuoespaciales (5). Puede administrarse en un tiempo relativamente breve ( 15 a 20 minutos). Comparado con el MMSE, el ACE brinda más información en memoria, denominación y visuo-espacialidad, e incorpora además la medición de funciones ejecutivas (p. ej., fluencia verbal, prueba del reloj). Además, contiene en su estructura al MMSE. Como se mencionó previamente, del ACE puede extraerse un coeficiente denominado VLOM, (fluidez verbal + lenguaje) / (orientación + recuerdo diferido) diseñado para orientar la discriminación entre la DA y DFT.

El trabajo de Sarasola y col. validó el ACE en una población de habla hispana con alto nivel educativo (instrumento disponible en su la versión empleada por los autores de dicha validación en http://www.neurologiacognitiva.org/file/ACE.doc y en el cuadro 1). En ese escenario el instrumento mostró características operativas apropiadas para el diagnóstico de demencia leve. Además este mismo instrumento también ha sido validado en una población de habla hispana con menor nivel educativo ${ }^{10}$. En este caso el ACE mostró similares características operativas (ver tabla 1) utilizándose como punto de corte 68 . En otra publicación posterior, se demostró también que la capacidad diagnóstica del ACE no sufre influencias por estados depresivos concomitantes; es decir, la prueba discrimina igualmente a los pacientes con demencia, cumplan o no con criterios de depresión mayor ${ }^{11}$.

Con los puntos de corte elegidos el ACE gana sensibilidad respecto del MMSE, a expensas de una menor especificidad. Sin embargo estos datos en forma aislada dan una idea parcial de la utilidad real de la prueba. En un escenario teórico de baja prevalencia de enfermedad (5\%) el VPP* del ACE es algo menor que el del MMSE. Es decir, ante un resultado positivo de ambas pruebas, será más probable encontrar pacientes dementes en aquellos pacientes con MMSE positivo. En este mismo escenario ambas pruebas descartan razonablemente el diagnostico de demencia ante resultados negativos (alto VPN* en ambos casos). Pero cuando la prevalencia de enfermedad es mayor, los VPP* de ambas pruebas tienden a solaparse, y encontramos mayores diferencias en el VPN*. Así será más 
probable descartar la enfermedad en un paciente con ACE negativo que en uno con MMSE negativo.

Probablemente la mayor utilidad del ACE en el diagnóstico de demencia leve (como alternativa de prueba cognitiva breve) resida en descartar enfermedad en pacientes con resultados negativos, sobre todo cuando las prevalencias teóricas (o sospecha) de enfermedad son más altas. Sin embargo, no hay que perder de vista que el ACE pretende ser una herramienta breve de aplicación en ámbitos no especializados y sin embargo fue validada en un escenario diferente al de la atención primaria; siendo esta limitación muy frecuente a la hora de contar con evaluaciones de este tipo de prueba.

\section{Comentario final}

El proceso diagnóstico del deterioro cognitivo y demencia es complejo, pues implica la adquisición de conocimientos y competencias que van más allá de la aplicación de una prueba de evaluación cognitiva . La creciente aparición de clínicas especializadas de evaluación cognitiva da cuenta de esta realidad y probablemente también reflejen un aumento de la demanda de la población preocupada por problemas de memoria. Sin embargo también es cierto que en la realidad de la atención primaria estos recursos no siempre están disponibles y que muchos casos de deterioro cognitivo pasan por alto hasta que el diagnóstico es evidente a simple vista. En esta instancia probablemente el médico de cabecera pueda formular un diagnostico con mayor comodidad y no requiera de una evaluación especializada. Sin embargo en los escenarios donde la incertidumbre diagnóstica es mayor (diagnóstico diferencial entre anciano cognitivamente normal, deterioro cognitivo leve o demencia leve) es posible que el uso de pruebas cognitivas breves como las que se mencionan, pueda colaborar a la mejor selección de los pacientes que deban ser consultados con los especialistas (geriatras, neurólogos o psiquiatras) para definir un diagnostico preciso, trascendente tanto para el paciente como para su familia.

Ver glosario*

Recibido el 10/02/2009 y aceptado el 10 de marzo del 2009

\section{Referencias}

1. Ferri C. y col. Global prevalence of dementia: a Delphy consensus study. Lancet 2005;366:2112-17. Disponible en: http://www.sbgg.org.br/profissional/artigos/pdf/demencia_mundo.pdf (último acceso 22/02/2009).

2. Arizaga R. y col. Deterioro cognitivo en mayores de 60 años en cañuelas (Argentina). Resultados del piloto del estudio ceibo (estudio epidemiológico poblacional de demencia) [resumen]. Rev Neurol Arg 2005;30(2):83-90. Disponible en:

http://www.imbiomed.com.mx/1/1/articulos.php?method=showDetail\&id_articulo=37246\&id_seccion=1257\&id_ejemplar=3845\&id_revista=95. (último acceso 22/02/2009)

3. Centurión E. y col. Resumen ejecutivo: Estado de conocimiento y agenda de prioridades para la toma de decisiones en enfermedades neurológicas: Alzheimer y otras demencias en Argentina. Informe perteneciente al Foro de Investigación en Salud de Argentina. Buenos Aires: Cámara Argentina del Libro; 2008. Disponible en: http://www.saludinvestiga.org.ar/pdf/policy-brief/2008-alzheimer.pdf (último acceso 22/02/2009)

4. INDEC. Censo Nacional de Población, Hogares y Viviendas 2001 [en línea]. Disponible en: http://www.indec.mecon.gov.ar/principal.asp?id_tema=50 (último acceso 22/02/2009).

5. U.S. Preventive Services Task Force. Screening for dementia. Recommendations and rationale (en linea). Rockville, 2003. Disponible en: http://www.ahrq.gov/clinic/3rduspstf/dementia/dementrr.pdf (último acceso 10/03/2009).

6. Howard H, y col. Diagnosis and treatment of dementia: 2 diagnosis. CMAJ 2008;178(7):825-36.

7. Holsinger T, y col. Does This Patient Have Dementia?. JAMA 2007;297(21):2391-2404.

8. Sarasola y col. El Addenbrooke's Cognitive Examination en español para el diagnóstico de demencia y para la diferenciación entre enfermedad de Alzheimer y demencia frontotemporal. Rev. Neurol 2005;41(12):717-21

9. Mathuranath $\mathrm{P}, \mathrm{y}$ col. A brief cognitive test battery to differentiate Alzheimer's disease and frontotemporal dementia. Neurology 2000;11(55).

10. García Caballero A, y col. Validation of the Spanish version of the Addenbrooke's Cognitive Examination in a rural community in Spain. Int J Geriatr Psychiatry 2006:21:239245

11. Roca M, y col. Diferenciación entre demencias en estadío inicial y depresión utilizando la versión española del Addenbrooke's Cognitive Examination. Rev Neurol 2008;46(6):340-43

12. Queja cognitiva y demencia. En: PROFAM, Salud del Anciano. Rubinstein E, et al Editores. 3ra edición. Buenos Aires: Fundación MF "Para el desarrollo de la Medicina Familiar y la Atención primaria de la salud", 2006. 


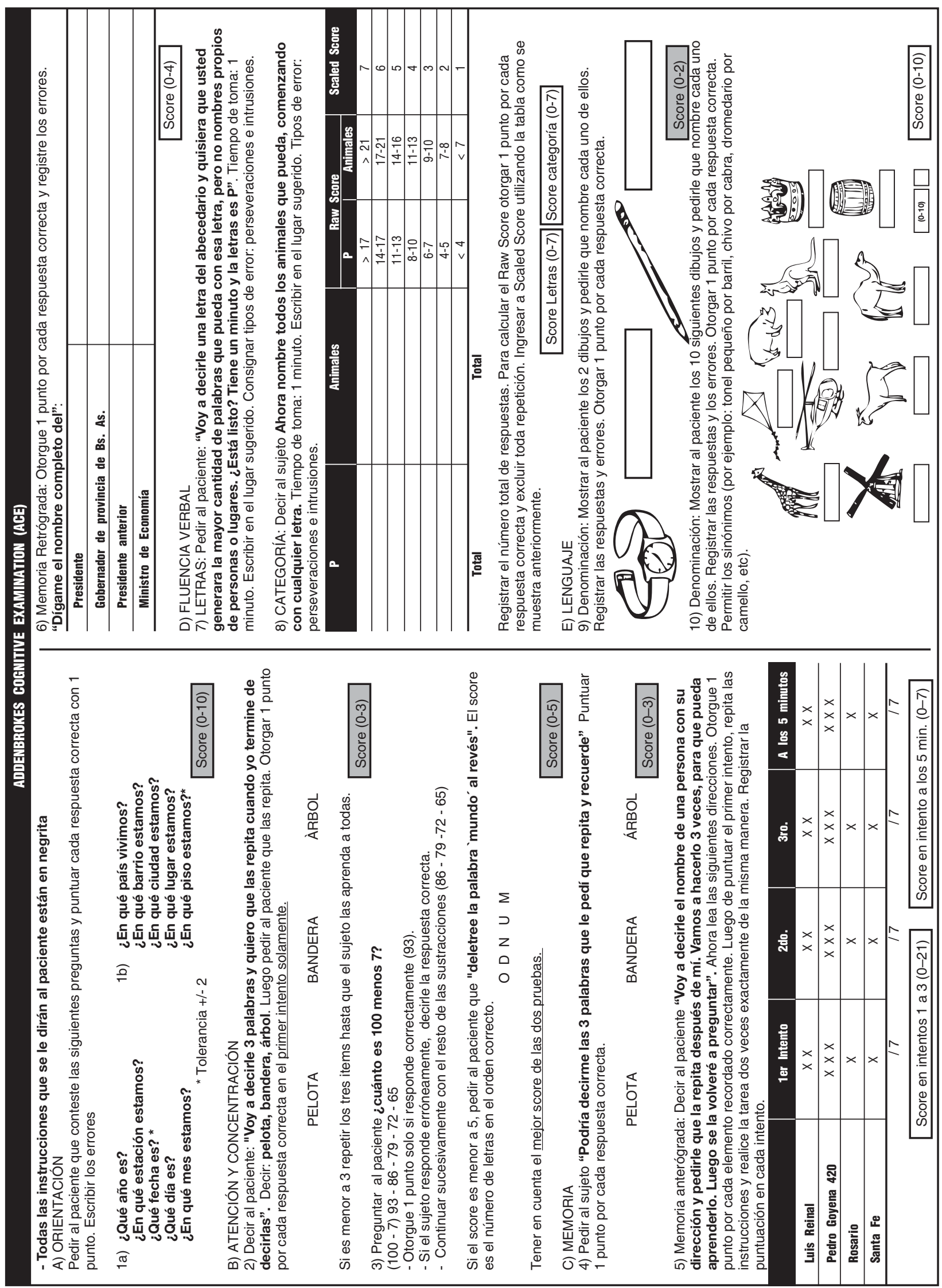




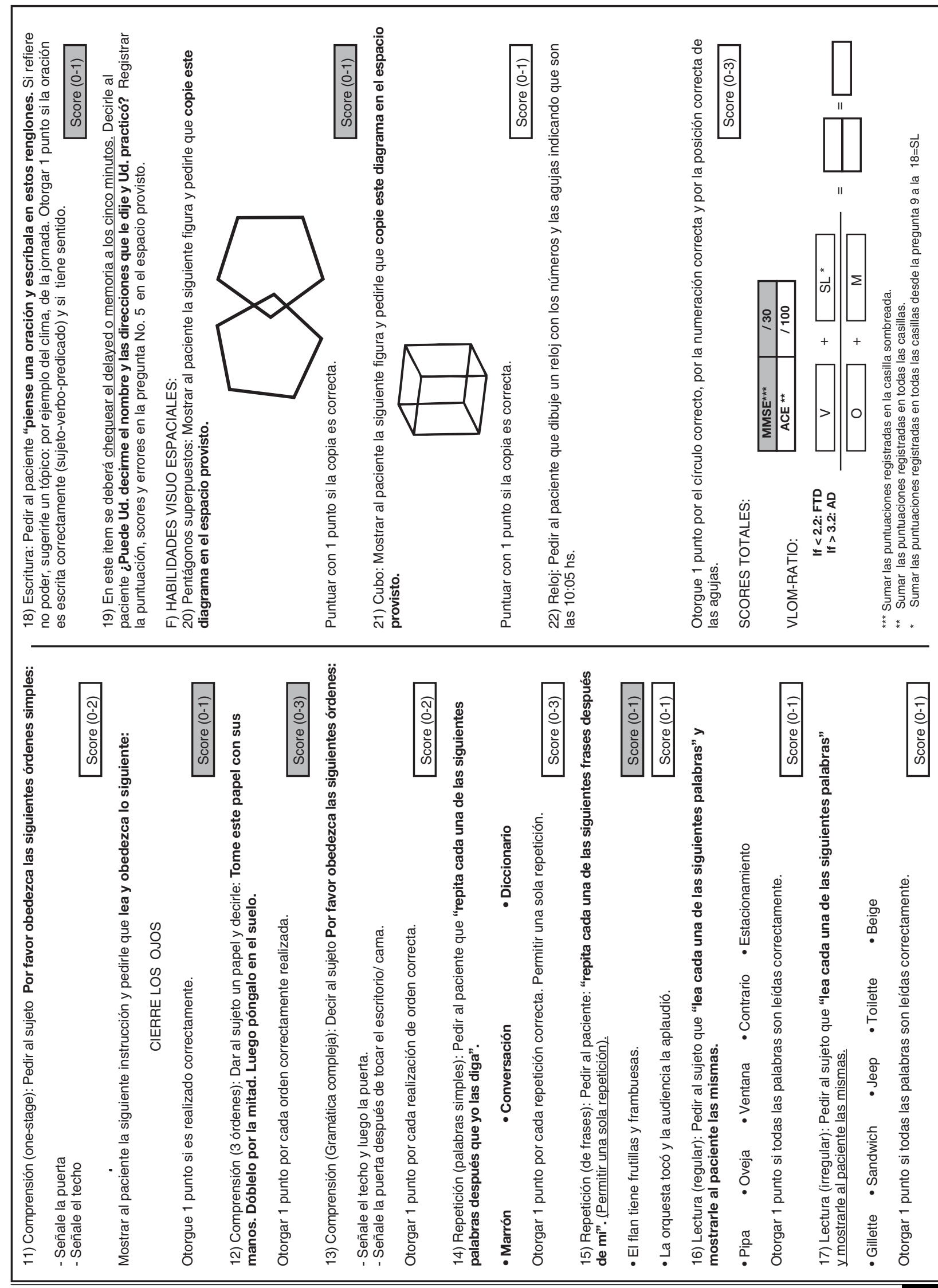

